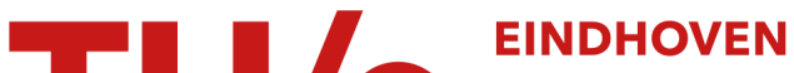 UNIVERSITY OF TECHNOLOGY
}

\section{Duality Symmetry in Hybrid Nanoresonators for Chiral Sensing}

Citation for published version (APA):

Mohammadi, E., Tittl, A., Tsakmakidis, K. L., Raziman, T. V., \& Curto, A. G. (2020). Duality Symmetry in Hybrid Nanoresonators for Chiral Sensing. In B. Lee, C. Mazzali, \& K. Corwin (Eds.), Frontiers in Optics - Proceedings Frontiers in Optics / Laser Science, Part of Frontiers in Optics + Laser Science APS/DLS, FiO 2020: Nanophotonics and Nanoplasmonics [FTh5D.8] Optical Society of America (OSA). https://doi.org/10.1364/FIO.2020.FTh5D.8

\section{Document license:}

Unspecified

DOI:

10.1364/FIO.2020.FTh5D.8

Document status and date:

Published: 17/09/2020

\section{Document Version:}

Accepted manuscript including changes made at the peer-review stage

\section{Please check the document version of this publication:}

- A submitted manuscript is the version of the article upon submission and before peer-review. There can be important differences between the submitted version and the official published version of record. People interested in the research are advised to contact the author for the final version of the publication, or visit the $\mathrm{DOI}$ to the publisher's website.

- The final author version and the galley proof are versions of the publication after peer review.

- The final published version features the final layout of the paper including the volume, issue and page numbers.

Link to publication

\section{General rights}

Copyright and moral rights for the publications made accessible in the public portal are retained by the authors and/or other copyright owners and it is a condition of accessing publications that users recognise and abide by the legal requirements associated with these rights.

- Users may download and print one copy of any publication from the public portal for the purpose of private study or research.

- You may not further distribute the material or use it for any profit-making activity or commercial gain

- You may freely distribute the URL identifying the publication in the public portal.

If the publication is distributed under the terms of Article 25fa of the Dutch Copyright Act, indicated by the "Taverne" license above, please follow below link for the End User Agreement:

www.tue.nl/taverne

Take down policy

If you believe that this document breaches copyright please contact us at:

openaccess@tue.nl

providing details and we will investigate your claim. 


\title{
Duality Symmetry in Hybrid Nanoresonators for Chiral Sensing
}

\author{
Ershad Mohammadi ${ }^{1}$, Andreas Tittl ${ }^{2}$, Kosmas L. Tsakmakidis ${ }^{3}$, T.V. Raziman ${ }^{1}$, \\ Alberto G. Curto ${ }^{1}$ \\ ${ }^{1}$ Department of Applied Physics and Institute for Photonic Integration, Eindhoven University of Technology, \\ Eindhoven, The Netherlands. ${ }^{2}$ \\ Chair in Hybrid Nanosystems, Nanoinstitute Munich, Faculty of Physics, Ludwig-Maximilians-Universität \\ München, 80539, Munich, Germany. \\ ${ }^{3}$ Section of Condensed Matter Physics, Department of Physics, National and Kapodistrian University of Athens, \\ Panepistimioupolis, GR-157 84 Athens, Greece \\ e.mohammadi@tue.nl
}

\begin{abstract}
Metal nanoparticles support intense electric resonances, while high-index dielectric particles offer strong magnetic resonances. Here, we propose metal-dielectric nanophotonic platforms based on duality symmetry for chiral molecular sensing. (C) 2020 The Author(s)
\end{abstract}

\section{Introduction}

Chirality is vital for the functionality of biomolecules and, consequently, for the interactions of chiral drugs with the human body. The detection and differentiation of chiral molecules is thus of great interest in fields ranging from biology and pharmacy to the cosmetics and food industries. Chiral molecules reveal their handedness through interaction with another chiral entity, such as circularly polarized light. Circular dichroism spectroscopy exploits the differential absorption of chiral samples for right- and left-handed circular polarizations. According to Poynting's theorem, this difference in absorbed power for a chiral sample that is illuminated sequentially with right $(+)$ and left(-) circularly polarized light can be expressed as [1]:

$$
P_{+}-P_{-}=4 c_{0} \operatorname{Im}\{\kappa\} C V_{s}
$$

where $c_{0}, \kappa$ and $V_{s}$ are the speed of light, the Pasteur parameter of the chiral sample [2], and the sample volume, respectively. $C$ represents the optical chirality [3]. The differential absorbed power in eq. (1) is normally exceptionally weak because the Pasteur parameter for biomolecules is extremely small [4] $\left(\kappa \sim 10^{-4}-10^{-7}\right)$. Furthermore, a general goal is to push the detection limits down to very small volumes of chiral sample for maximum sensitivity. Interestingly, these weak signals can be compensated by an increase in the optical chirality of the field interacting with the sample, which motivates the quest to find nanophotonic platforms with the highest values of $C[5,6]$.

For superchiral field formation, we need electric and magnetic fields that are parallel, $\pi / 2$ out of phase, and with spectral and spatial overlap. Metallic nanostructures can create intense electric dipole fields through the excitation of localized surface plasmon resonances. On the other hand, high-refractive-index dielectric nanoparticles can exhibit strong magnetic dipole resonances [7-9]. This complementarity opens a hybrid route to exploit both types of resonances for enhancing optical chirality.

Here, we propose a combination of metal and dielectric structures capable of providing (i) strong electric and magnetic fields as well as (ii) equal electric and magnetic response, known as duality symmetry [10]. The latter brings the electric and magnetic resonances to the same spectral and spatial range and satisfies $\pi / 2$ phase condition. Furthermore, our hybrid nanostructures offer a degree of freedom in design by decoupling and separately controlling the electric and magnetic response, The concept of duality symmetry (Figure 1a) relies on the electric dipolar field $\left(\mathbf{E}_{\text {dip }}\right)$ of an electric resonator excited by the incident electric field $\left(\mathbf{E}_{\text {inc }}\right)$ being analogous to the magnetic dipolar field $\left(\mathbf{H}_{\text {dip }}\right)$ of a magnetic resonator excited by the incident magnetic field $\left(\mathbf{H}_{i n c}\right)$. To harness the dual fields of such individual resonators in a nanophotonic system, we first focus on nanodimers to analytically elucidate how duality symmetry can be achieved. Then, to realize strong dual fields with realistic materials, we propose a hybrid metal-dielectric dimer system (Figure 1b) composed of a high-refractive-index dielectric particle placed next to a plasmonic particle. Based on the insights gained from simple nanodimers, we introduce a more complex hybrid metasurface for chiral sensing enabling a 350-fold optical chirality enhancement. The spatially averaged optical chirality is high, resulting in an enhancement of circular dichroism by a factor of 20 .

Our theoretical approach shows thus that this route meets the two fundamental requirements for attaining optimal superchiral fields. By combining individually tunable resonators, our hybrid structures offer a design strategy 


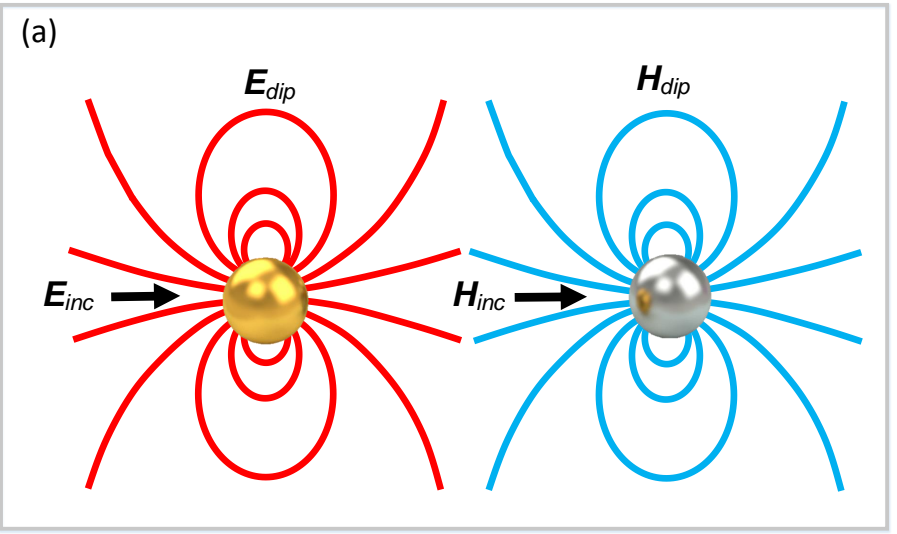

(b)

Fig. 1. (a) Concept of duality symmetry to realize superchiral fields. The dual electric (yellow sphere) and magnetic (grey sphere) resonators show analogous electric and magnetic fields that can be used for achieving strong, parallel,spectrally and spatially overlapped and finally $\pi / 2$ out of phase electric and magnetic fields. $\mathbf{E}_{d i p}$ (red) and $\mathbf{H}_{\text {dip }}$ (blue) indicate the dual dipolar fields associated with electric and magnetic resonators. (b) A hybrid metal-dielectric dimer illuminated by circularly polarized light producing strong optical chirality. The strong electric resonance in the plasmonic particle is coupled to the electric resonance of the dielectric particle forming a strong electric hotspot that is fully decoupled from the strong magnetic resonance of the dielectric sphere. The duality symmetry of the structure provides a phase factor of $\pi / 2$ between the electric and magnetic hotspots. Red and blue helices indicate the electric and magnetic fields, respectively.

\section{References}

1. Ershad Mohammadi, Ahad Tavakoli, Parisa Dehkhoda, Yasaman Jahani, Kosmas L Tsakmakidis, Andreas Tittl, and Hatice Altug. Accessible superchiral near-fields driven by tailored electric and magnetic resonances in all-dielectric nanostructures. ACS Photonics, 6(8):1939-1946, 2019.

2. Ismo V Lindell, AH Sihvola, SA Tretyakov, and AJ Viitanen. Electromagnetic waves in chiral and biisotropic media. Artech House Boston, 1994.

3. Yiqiao Tang and Adam E Cohen. Optical chirality and its interaction with matter. Physical Review Letters, 104(16):163901, 2010.

4. Zhiyuan Fan and Alexander O Govorov. Chiral nanocrystals: plasmonic spectra and circular dichroism. Nano Letters, 12(6):3283-3289, 2012.

5. Ventsislav K Valev, Jeremy J Baumberg, Concita Sibilia, and Thierry Verbiest. Chirality and chiroptical effects in plasmonic nanostructures: fundamentals, recent progress, and outlook. Advanced Materials, 25(18):2517-2534, 2013.

6. Euan Hendry, RV Mikhaylovskiy, LD Barron, Malcolm Kadodwala, and TJ Davis. Chiral electromagnetic fields generated by arrays of nanoslits. Nano Letters, 12(7):3640-3644, 2012.

7. Aitzol García-Etxarri, R Gómez-Medina, Luis S Froufe-Pérez, Cefe López, L Chantada, Frank Scheffold, J Aizpurua, M Nieto-Vesperinas, and Juan José Sáenz. Strong magnetic response of submicron silicon particles in the infrared. Optics Express, 19(6):4815-4826, 2011.

8. E Mohammadi, KL Tsakmakidis, AN Askarpour, P Dehkhoda, A Tavakoli, and H Altug. Nanophotonic platforms for enhanced chiral sensing. ACS Photonics, 5(7):2669-2675, 2018.

9. TV Raziman, Rasmus H Godiksen, Moos A Muller, and Alberto G Curto. Conditions for enhancing chiral nanophotonics near achiral nanoparticles. ACS Photonics, 6(10):2583-2589, 2019.

10. Ivan Fernandez-Corbaton, Xavier Zambrana-Puyalto, Nora Tischler, Xavier Vidal, Mathieu L Juan, and Gabriel Molina-Terriza. Electromagnetic duality symmetry and helicity conservation for the macroscopic maxwell's equations. Physical Review Letters, 111(6):060401, 2013. 